\title{
On the nature of singularities in plane symmetric scalar field cosmologies
}

\author{
Alan D. Rendall ${ }^{1}$
}

\begin{abstract}
The nature of the initial singularity in spatially compact plane symmetric scalar field cosmologies is investigated. It is shown that this singularity is crushing and velocity dominated and that the Kretschmann scalar diverges uniformly as it is approached. The last fact means in particular that a maximal globally hyperbolic spacetime in this class cannot be extended towards the past through a Cauchy horizon. A subclass of these spacetimes is identified for which the singularity is isotropic.
\end{abstract}

\section{INTRODUCTION}

The nature of singularities in general solutions of the Einstein equations is a subject about which much remains to be learned. Various classes of singularities have been defined which represent possible models for general behaviour. Examples are curvature singularities, crushing singularities[1], velocity dominated singularities[2] and isotropic singularities[3]. In this paper spacetimes belonging to one of the simplest classes of inhomogeneous cosmologies will be examined in order to get as much information as possible about their singularities and to test the applicability of the models just mentioned.

The spacetimes considered in the following are solutions of the Einstein equations coupled to a massless scalar field in the standard way. Thus, if $\phi$ denotes the scalar field they are solutions of

$$
G_{\alpha \beta}=8 \pi\left[\nabla_{\alpha} \phi \nabla_{\beta} \phi-\frac{1}{2}\left(\nabla^{\gamma} \phi \nabla_{\gamma} \phi\right) g_{\alpha \beta}\right]
$$

The Bianchi identities imply that $\phi$ satisfies the wave equation. These spacetimes are further assumed to be plane symmetric. Plane symmetric solutions of the Einstein equations with a scalar field as matter source have been discussed by Tabensky and Taub [4]. In fact their paper is on stiff fluids but, as they show, it is possible to transform between

1 Max-Planck-Institut für Astrophysik, Karl-Schwarzschildstr. 1, Postfach 1523, 85740 Garching, Germany. 
these two matter models under rather general circumstances. They write the field equations in a particularly simple form. If the gradient of the area of the orbits is everywhere timelike then these equations can be simplified further. This condition will be assumed in the following. It has been shown elsewhere that for appropriate boundary conditions it is automatically fulfilled unless the spacetime is flat[5]. Tabensky and Taub show that the only non-trivial equation to be solved is the linear hyperbolic equation

$$
\phi_{t t}+t^{-1} \phi_{t}=\phi_{x x}
$$

When this has been done a quantity $\Omega$ is obtained by integrating the ordinary differential equation

$$
\Omega_{t}=t\left(\phi_{t}^{2}+\phi_{x}^{2}\right)
$$

This can be done starting on an initial hypersurface of constant $t$. In order that all Einstein equations should be satified the constraint equation

$$
\Omega_{x}=2 t \phi_{t} \phi_{x}
$$

should hold on the initial hypersurface. The spacetime metric is

$$
d s^{2}=t^{-1 / 2} e^{\Omega}\left(-d t^{2}+d x^{2}\right)+t\left(d y^{2}+d z^{2}\right)
$$

Here $t$ belongs to the interval $(0, \infty)$. To avoid spurious singularities it is assumed that the spacetime is spatially compact. This can be arranged by demanding that the coordinates $x, y$ and $z$ be periodic. The periodicity of $y$ and $z$ plays no significant role in the following but the periodicity of $x$ means that $\phi$ and $\Omega$ (which only depend on $t$ and $x$ ) are required to be periodic in $x$.

The initial value problem for data given on the hypersurface $t=t_{0}>0$ can be solved as follows. An initial data set consists of periodic functions $\phi_{0}, \phi_{1}$ and $\Omega_{0}$ which satisfy the equation

$$
\left(\Omega_{0}\right)_{x}=2 t_{0} \phi_{1}\left(\phi_{0}\right)_{x}
$$

For simplicity they will be assumed to be $C^{\infty}$ although the arguments which follow can also be carried through when these functions have an appropriate finite degree of differentiability. A solution is sought with $\phi\left(t_{0}, x\right)=\phi_{0}(x), \phi_{t}\left(t_{0}, x\right)=\phi_{1}(x)$ and $\Omega\left(t_{0}, x\right)=\Omega_{0}(x)$. Under these conditions (6) is just the constraint equation (4) on the hypersurface $t=t_{0}$. To construct the solution first solve the linear hyperbolic equation (2) on the time interval $(0, \infty)$ with initial data $\phi_{0}$ and $\phi_{1}$. Standard theory ensures the existence of a unique $C^{\infty}$ solution $\phi$. Then $\Omega$ may be determined by integrating (3) with initial value $\Omega_{0}$ for each fixed value of $x$.

The solutions of the initial value problem have an apparent singularity at $t=0$. The aim of the following is to show that this is a true singularity (i.e. that the spacetime cannot be extended through it) and to obtain more detailed information about its nature. In Section 2 it is shown that $t=0$ is always a curvature singularity and that the Kretschmann scalar $R^{\alpha \beta \gamma \delta} R_{\alpha \beta \gamma \delta}$ blows up uniformly as $t \rightarrow 0$. The consequences for strong cosmic censorship are discussed. In Section 3 the singularity is shown to be crushing and it 
is concluded that a neighbourhood of the singularity can be foliated by constant mean curvature hypersurfaces. An asymptotic expansion for the solution in a neighbourhood of $t=0$ is obtained in Section 4 which shows in particular that the singularity is velocity dominated. In the final section a sufficient condition is given for the singularity to be isotropic.

Note that the simplification of the field equations which allows the analysis which follows to be carried out depends very much on the plane symmetry and the fact that the matter content of spacetime is described by a massless scalar field. If plane symmetry is replaced by spherical symmetry or if the massless scalar field is replaced by almost any other kind of matter, then the equations for the matter fields, the equation for $\Omega$ and the equation for the area of the orbits are all coupled. The property of the matter which is needed for decoupling is that the trace of the projection of the energy-momentum tensor to the orthogonal complement of the orbits should vanish.

\section{CURVATURE SINGULARITIES}

The curvature of a general plane-symmetric spacetime will now be computed. It is always possible to introduce local coordinates so that the metric takes the form

$$
d s^{2}=g_{a b} d x^{a} d x^{b}+r^{2} \delta_{A B} d y^{A} d y^{B}
$$

Here lower and upper case indices take the values 0,1 and 2,3 respectively. Let $K$ denote the Gaussian curvature of the two-dimensional metric $g_{a b}$ and let $\nabla_{a}$ denote the covariant derivative associated to that metric. Then the curvature components are

$$
\begin{aligned}
R_{b c d}^{a} & =K\left(\delta_{c}^{a} g_{b d}-\delta_{d}^{a} g_{b c}\right) \\
R_{B C D}^{A} & =-\nabla^{a} r \nabla_{a} r\left(\delta_{C}^{A} \delta_{B D}-\delta_{D}^{A} \delta_{B C}\right) \\
R_{B c D}^{a} & =-r \nabla^{a} \nabla_{c} r \delta_{B D}
\end{aligned}
$$

Hence

$$
R^{\alpha \beta \gamma \delta} R_{\alpha \beta \gamma \delta}=4 K^{2}+4 r^{-4}\left(\nabla^{a} r \nabla_{a} r\right)^{2}+8 r^{-2} \nabla_{a} \nabla_{b} r \nabla^{a} \nabla^{b} r
$$

When the curvature components have been computed the Einstein equations can easily be obtained. One combination of the field equations gives

$$
\nabla_{a} \nabla_{b} r=-\frac{1}{2 r} \nabla^{c} r \nabla_{c} r g_{a b}-4 \pi r\left(T_{a b}-\operatorname{tr} \mathrm{T} g_{a b}\right)
$$

where $\operatorname{tr} T=g^{a b} T_{a b}$. Combining (11) and (12):

$$
\begin{aligned}
R^{\alpha \beta \gamma \delta} R_{\alpha \beta \gamma \delta}=4 & K^{2}+4 r^{-4}\left(\nabla^{a} r \nabla_{a} r\right)^{2}+r^{-4}\left(\frac{1}{2 r} \nabla^{c} r \nabla_{c} r-2 \pi r \operatorname{tr} \mathrm{T}\right)^{2} \\
+ & 16 \pi^{2} r^{-2}\left(T_{a b}-\frac{1}{2} \operatorname{trT} g_{a b}\right)\left(T^{a b}-\frac{1}{2} \operatorname{trT} g^{a b}\right)
\end{aligned}
$$


The first three terms on the right hand side of (13) are obviously non-negative and when the matter content of spacetime is described by a massless scalar field the fourth term is non-negative. (This condition also holds for many other physically reasonable matter fields but that fact is not relevant for this paper.) It follows that if $m=-r \nabla_{a} r \nabla^{a} r / 2$ then

$$
R^{\alpha \beta \gamma \delta} R_{\alpha \beta \gamma \delta} \geq 16 m^{2} / r^{6}
$$

Returning from these general considerations to the particular class of spacetimes considered here, it turns out that in that case $m=\frac{1}{8} e^{-\Omega}$ and $r$ is a constant times $t^{1 / 2}$. It follows from (3) that $\Omega$ is non-decreasing. Hence the curvature invariant $R^{\alpha \beta \gamma \delta} R_{\alpha \beta \gamma \delta}$ blows up at least as fast as $t^{-3}$ as $t=0$ is approached.

It has now been shown that $t=0$ is a curvature singularity and thus the spacetime cannot be extended further. This gives a statement which might be called 'strong cosmic censorship in the past' for the class of spacetimes considered here. (This terminology assumed that a time orientation of spacetime has been chosen so that $t$ increases towards the future.) It says that if a spacetime of this type is the maximal globally hyperbolic development of initial data on some hypersurface then no extension of that spacetime contains a point to the past of the initial hypersurface which does not belong to the original spacetime.

\section{CRUSHING SINGULARITIES}

The mean curvature of the hypersurfaces of constant $t$ is given by

$$
H=-\frac{1}{2} t^{1 / 4} e^{-\Omega / 2}\left(\Omega_{t}+\frac{3}{2 t}\right)
$$

Equation (3) shows that $\Omega_{t} \geq 0$ and so (15) implies that $|H| \geq \frac{3}{4} t^{-3 / 4} e^{-\Omega / 2} \geq C t^{-3 / 4}$. Thus it can be seen that $H$ tends uniformly to $-\infty$ as the singularity is approached. This means that this singularity is a crushing singularity[1]. A crushing singularity in a spatially compact spacetime always has a neighbourhood which can be foliated by hypersurfaces of constant mean curvature. The proof of this will now be recalled. Note first that a spacetime which has a compact Cauchy hypersurface can contain at most one compact spacelike hypersurface with a given non-zero constant mean curvature[6]. The fact that $|H|$ tends uniformly to infinity shows that given any real number $H_{1}$ which is sufficiently large and negative there exist $t_{1}, t_{2}>0$ such that the hypersurface $t=t_{1}$ has mean curvature less than $H_{1}$ and the hypersurface $t=t_{2}$ has mean curvature greater than $H_{1}$. These hypersurfaces provide barriers which ensure that there exists a hypersurface of constant mean curvature $H_{1}$ between the hypersurfaces of $t=t_{1}$ and $t=t_{2}[7,8]$. Thus there is an interval $\left(-\infty, H_{0}\right]$ such that the spacetime contains exactly one compact hypersurface of constant mean curvature $H_{1}$ for each $H_{1}$ in this interval. It remains to show that these hypersurfaces cover a neighbourhood of the singularity. A standard result[6] implies that if $H_{2}<H_{1}$ the hypersurface of mean curvature $H_{2}$ lies strictly to the past of that with mean curvature $H_{1}$. By construction the hypersufaces tend to the singularity as $H_{1} \rightarrow-\infty$. In

other words there is no point which lies to the past of all these hypersurfaces. It remains to show that there are no gaps, i.e. that there is no point which lies to the past of the 
hypersurface with mean curvature $H_{1}$ but to the future of the hypersurfaces with mean curvature $H_{2}$ for all $H_{2}<H_{1}$. Suppose that a point $p$ with this property existed. Then there would be an open neighbourhood $U$ of the hypersurface with mean curvature $H_{1}$ disjoint from the future of $p$. In $U$ there exist hypersurfaces of constant mean curvature $H_{2}$ for all $H_{2}$ in some interval $\left[H_{1}-\epsilon, H_{1}+\epsilon\right]$ with $\epsilon>0$. Hence there is a point of the hypersurface with mean curvature $H_{1}+\epsilon$ which lies to the past of the hypersurface with $H_{1}-\epsilon / 2$, contradicting a statement made earlier. It follows that no point $p$ with the above property can exist.

\section{VELOCITY DOMINATED SINGULARITIES}

The central problem in analysing the singularities in the class of spacetimes considered here is to determine the behaviour of a general spatially periodic solution of equation (2) as $t \rightarrow 0$. Letelier and Tabensky[9] have written down an integral formula for solutions of this equation but they give an explicit example of a solution which is not of that form. They conjecture that all solutions can be obtained as limits of solutions given by the integral formula. Without a proof of this conjecture their analysis is incomplete. This problem can be circumvented by the direct use of energy estimates, as has been shown by Isenberg and Moncrief[10] in the course of a study of polarised Gowdy spacetimes. A sketch of the argument will now be given. It will be supposed for simplicity that the solution $\phi$ being considered is $C^{\infty}$. A computation gives the inequality

$$
\frac{d}{d t} \int t^{2}\left(\phi_{t}^{2}+\phi_{x}^{2}\right) \geq 0
$$

for $t>0$ when $\phi$ is a solution of (2). Since the coefficients in the equation do not depend explicitly on the spatial coordinate the derivative of $\phi$ of any order with respect to $x$ satisfies the same equation as $\phi$ itself. Hence all spatial derivatives of $\phi$ satisfy inequalities analogous to (16). The Sobolev embedding theorem then implies that $t \phi_{t}, t \phi_{x}$ and the derivatives of these quantities with respect to $x$ of any order are bounded in a neighbourhood of $t=0$. Equation (2) can be rewritten as

$$
\left(t \phi_{t}\right)_{t}=t \phi_{x x}
$$

Knowing that $t \phi_{x x}$ is bounded allows us to conclude that $t \phi_{t}$ has a continuous extension to $t=0$. Integrating twice in time gives the asymptotic expansion

$$
\phi(t, x)=\pi(x) \log t+\omega(x)+O(t)
$$

for some smooth functions $\pi(x)$ and $\omega(x)$ as $t \rightarrow 0$. The expression obtained by formally differentiating (18) once with respect to $t$ and as many times as desired with respect to $x$ is also a valid asymptotic expansion. Substituting these asymptotic expansions into the evolution equation for $\Omega$ gives

$$
\Omega_{t}=\pi^{2}(x) t^{-1}+O(1)
$$

Integrating this with respect to $t$ gives

$$
\Omega(t, x)=\pi^{2}(x) \log t+\alpha(x)+O(t) .
$$


for some function $\alpha$. Let the parts of the right hand sides of (18) and (20) explicitly written out be denoted by $\tilde{\phi}$ and $\tilde{\Omega}$ respectively so that $\phi=\tilde{\phi}+O(t)$ and $\Omega=\tilde{\Omega}+O(t)$. The quantities $\tilde{\phi}$ and $\tilde{\Omega}$ are solutions of the equations obtained from the Einstein evolution equations by dropping all spatial derivatives. This is what Isenberg and Moncrief [10] call the velocity dominated system. Thus the solutions of the full Einstein equations are approximated asymptotically near the singularity by solutions of the velocity dominated system and these spacetimes have what Isenberg and Moncrief call the AVTDS property (asymptotically velocity-term dominated near the singularity). This is not literally the same as the original definition of velocity dominated singularities which was given by Eardley, Liang and Sachs [2] but the spirit is the same and so for brevity this property is described here as the property that the singularity is velocity dominated.

The definition of a velocity dominated singularity makes use of a preferred foliation by spacelike hypersurfaces. A singularity which has the velocity dominated property with respect to one foliation will in general not have it with respect to a different foliation. In the present case it has been shown that the property holds with respect to the foliation defined by the time coordinate $t$ and this could be interpreted as saying that this foliation is in some sense well-behaved near the singularity. Taking this view it is natural to ask whether the foliation by hypersurfaces of constant mean curvature, whose existence was shown in Sect. 3, is also well-behaved in this sense. Despite the excellent control over the spacetime which is available, this question appears difficult to decide. It would be interesting to know the answer for the following reason. The time coordinate $t$ is defined in terms of the symmetry of the solution and so has no obvious analogue in general spacetimes with less symmetry. On the other hand the constant mean curvature condition makes sense in any spacetime and it seems reasonable to hope that foliations of constant mean curvature exist in a wide class of spacetimes.

\section{ISOTROPIC SINGULARITIES}

In the literature there has been some discussion of isotropic singularities, a class of singularities which is of relevance to Penrose's Weyl curvature hypothesis. A singularity of this kind can be defined $[3,11]$ by the condition that it should be possible to conformally rescale the given metric so that the rescaled metric extends regularly through the singularity. In general the asymptotic form of the spacetime metric near the singularity in the class of spacetimes considered here is

$$
t\left[t^{\pi^{2}-3 / 2} e^{\alpha}\left(-d t^{2}+d x^{2}\right)+\left(d y^{2}+d z^{2}\right)\right]
$$

This shows that if $\pi^{2}=3 / 2$ everywhere the conformal class of the metric extends continuously to $t=0$. Thus the singularity is isotropic in this case.

In fact it is desirable to require a little more of an isotropic singularity than what has just been demonstrated. The conformally rescaled metric should extend not just in a continuous non-degenerate manner to the singularity. It should also have some degree

of differentiability there. This question of the differentiability of the rescaled metric (or more precisely the question of the simultaneous differentiability of the rescaled metric and 
the conformal factor) is in general somewhat subtle [11]. However it turns out that in the present case everything can be made $C^{\infty}$. To show this it is necessary to extend the asymptotic expansions (17) and (19) to all powers of $t$. An asymptotic expansion of this type for the solution of (17) has been given in [12]. This is an expansion in integral powers of $t$ and $\log t$. However, if $\pi$ is constant the only term containing a logarithm is that written out explicitly in (17). It follows immediately that in the case $\pi=$ const. (19) can be extended to an asymptotic expansion to all orders which except for the first term is an expansion in integral powers of $t$. This shows that the rescaled metric is $C^{\infty}$ in the case identified as being isotropic above.

\section{REFERENCES}

1. Eardley, D., Smarr, L. (1979) Time functions in numerical relativity: marginally bound dust collapse. Phys. Rev. D19, 2239-2259.

2. Eardley, D., Liang, E. and Sachs, R. (1972) Velocity-dominated singularities in irrotational dust cosmologies. J. Math. Phys. 13, 99-106.

3. Goode, S., Wainwright, J. (1985) Isotropic singularities in cosmological models. Class. Quantum Grav. 2, 99-115.

4. Tabensky, R., Taub, A. H. (1973) Plane symmetric self-gravitating fluids with pressure equal to energy density. Commun. Math. Phys. 29, 61-77.

5. Rendall, A.D. Crushing singularities in spacetimes with spherical and plane symmetry. Unpublished.

6. Marsden, J., Tipler, F. (1980) Maximal hypersurfaces and surfaces of constant mean curvature in general relativity. Phys. Rep. 66, 109-139.

7. Gerhardt, C. (1983) H-surfaces in Lorentzian manifolds. Commun. Math. Phys. 89, 523-553.

8. Bartnik, R. (1984) Existence of maximal hypersurfaces in asymptotically flat spacetimes. Commun. Math. Phys. 94, 155-175.

9. Letelier, P., Tabensky, R. (1975) Singularities for fluids with $p=\omega$ equation of state. J. Math. Phys 16, 8-10.

10. Isenberg, J., Moncrief, V. (1990) Asymptotic behaviour of the gravitational field and the nature of singularities in Gowdy spacetimes. Ann. Phys. 199, 84-122.

11. Tod, K. P. (1992) Isotropic singularities. Rend. Sem. Mat. Univers. Politecn. Torino 50, 69-92.

12. Chruściel, P. T., Isenberg, J. and Moncrief, V. (1990) Strong cosmic censorship in polarised Gowdy spacetimes. Class. Quantum Grav. 7, 1671-1680. 